\title{
Battery Monitoring and Power Management for Automotive Systems
}

\author{
Anila Thyagarajan*, R. Raja Prabu, G. Uma \\ B.S Abdur Rahman University, India \\ *Corresponding author: anila1981@yahoo.com
}

Received March 17, 2013; Revised October 20, 2013; Accepted February 08, 2014

\begin{abstract}
New vehicle electric systems are promoted by the needs of fuel economy and ecology as well as by new functions for the improvement of safety and comfort, reliability, and the availability of the vehicle. Electrically controlled and powered systems for braking, steering and stabilization need a reliable supply of electrical energy. The planned generation of electrical energy (only when it is economically beneficial meaningful), an adequate storage, and thrifty energy housekeeping with an intelligent integration of the battery as the storage medium into the overall concept of the vehicle Energy Management, and early detection of possible restrictions of reliability by Battery Monitoring allows for actions by the Energy Management well in advance, while the driver need not be involved at all. To meet today's requirements for Battery Monitoring and Energy Management, solutions have been developed for series vehicles launched in years 2001-2003, operating at the $14 \mathrm{~V}$ level. In this paper, Battery Monitoring and Management has been done by considering the change of lead-acid battery properties over life, including grid corrosion, water loss, changes of active material properties, loss of active material availability, etc. Also, Battery Monitoring and Power Management to provide cranking capability several days ahead is also analyzed.
\end{abstract}

Keywords: automotive battery, SLI, vehicle electric power system, cattery monitoring, state-of-charge (SOC), state-of-health (SOH), battery management, energy management

Cite This Article: Anila Thyagarajan, R. Raja Prabu, and G. Uma, "Battery Monitoring and Power Management for Automotive Systems.” American Journal of Energy Research, vol. 2, no. 1 (2014): 1-8. doi: 10.12691/ajer-2-1-1.

\section{Introduction}

The term "Battery Monitoring" is used in a wide range of meanings, from occasional manual readings of voltages, of electrolyte gravity SG and level, and visual cell inspection, through periodical tests of capacity or manual measurement of battery resistance, to fully automated online supervision in critical applications with means for real-time estimation of residue bridging time, or of battery wear and tear.

In this paper, the term Battery Monitoring is used for supervision without manual engagement, which is stateof- the-art with many cycling batteries in automatically guided vehicles (AGVs), forklift trucks, submarines, electrically driven cars and trucks, as well as with standby batteries in telecom and UPS applications. With consumer applications, any mobile phone, laptop or pocket computer, or even a wristwatch is equipped with a device providing some information with respect to energy being left.

The specific different situation of automotive batteries becomes obvious, technically impeding Battery Monitoring in the automotive fields:

1. They are scarcely ever been completely charged, i.e. 'opportunity charge' is standard.

2. Recharge is performed with a wide range of different current rates.
3. Discharge virtually never starts from a full SOC.

4. Discharge is performed with a wide range of different current rates. Sometimes full discharge or (unfortunately) even over-discharge occurs.

5. Operational temperature may even exceed the window from 30 to 70 degrees

While the term "Battery Monitoring" comprises

1.taking and/or receiving data from and/or about the battery

2. processing of this information, including predictions of performance, and

3. being or a unit, i.e. only passive surveillance and evaluation

The term "Battery Management" means active feedback to the battery. This may comprise control of current or voltage levels, control of recharge conditions, limiting of the operational windows with respect to SOC and/or temperature, battery temperature management, etc.

An appropriate Battery Management may significantly enhance and improve, but is not a precondition for, a successful Energy Management. Figure 1 sketches the layer structure of Battery Monitoring generating Battery Status Information, Battery Management, and Energy Management.

It is Energy Management, preferably including Battery Management, which, based on the information from Battery Monitoring, allows for a self-standing operation of 
a system without manual input-the comfort and the technical necessity requested for a vehicle at the beginning of the $21^{\text {st }}$ century.

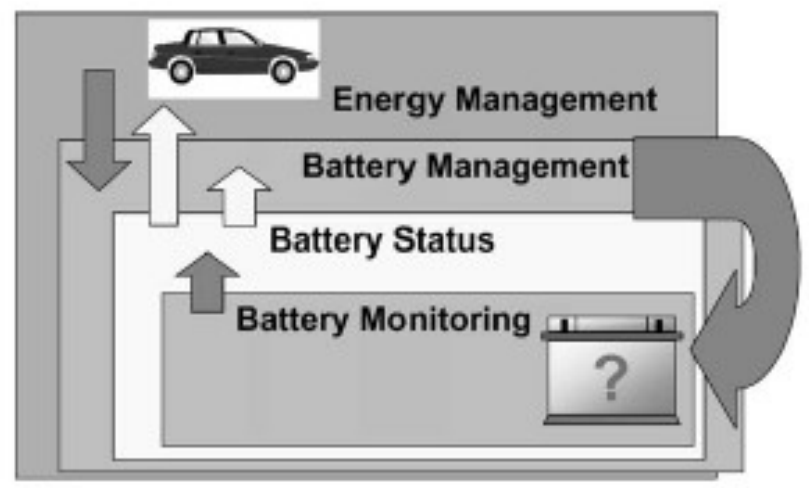

Figure 1. Layer structure of Battery Monitoring and Mutual Data Flow

\section{Vehicle Electric Power Systems}

Vehicle electric power systems are driven more and more by the needs of fuel economy and ecology as well as by new functions for the improvement of safety and comfort. New components may improve comfort and reliability, and the availability of the vehicle. In many cases, there is potential to reduce production and operational cost. Electrically controlled and powered systems for braking, steering and stabilisation need a reliable supply of electrical energy. Possible restrictions of reliability have to be prevented by the Energy Management and evaluated in advance, while the driver need not be involved at all.

Reduction of fuel consumption is expected to be achieved by replacement of mechanically driven auxiliary components by electrical components, which are been activated only when they are needed, and higher energy efficiency with generation, distribution, and use of electrical energy. While these goals are aiming at improvements of electrical engines, energy transfer and design of the electrical consumers, an important contribution can also be given by the planned generation of electrical energy, an adequate storage, and a thrifty energy housekeeping. Electric energy has to be generated when it is economically beneficial, and stored until it is needed in periods when generation is either inefficient or not possible at all.

This means an intelligent integration of the battery as the storage medium into the overall concept of the vehicle Energy Management. Careful monitoring and control of energy flows allows for minimum investment with respect to cost, weight and volume.

The overall requisite electrical performance is increasing with much higher fluctuations of the load demand than today. This cannot be covered by simple scaling up of today's components. Procedures are needed for optimal use of the battery resource: knowledge of actual state-of-charge, power capability, and degradation of the battery as an input for Energy Management.

A. Automotive Battery in the Past

In the beginning of the development of road vehicles driven by an internal combustion engine (ICE), there was no electrical equipment at all on board of the vehicle besides the ICE ignition, realized by magneto ignition or-more reliably-by primary dry cells. Lighting of luxury cars was soon provided electrically by storage batteries.

In view of the fact that the start routine is a very short one, both components, battery and starter motor, have, over the years, undergone a complete optimization to obtain the best possible torque for the lowest possible manufacturing costs. The further development of the vehicle electrical system was favored by the fact that increasingly powerful (claw- pole type) alternators became available at ever-decreasing manufacturing costs, and the vehicle battery, which was repeatedly called upon to provide cold starting power, was able to deliver some energy at all times to cover electrical requirements even during periods when the power supply to consumers was inadequate.

The dc alternators suffered from low or even no power output at low revs, so the battery had to provide electric power not only when the engine was at rest, but also when it was on idle. This was not an issue for decades, as electrical ignition, lighting and windscreen-wipers were the only consumers, and features like radio and electrically driven fans were limited to upper-class vehicles. In the 1960s, the automotive industry countered a major electrical energy bottleneck, caused by the rapid rise in the number of electrical consumers installed, esp. the introduction of the electrical window defroster, by doubling the battery voltage to $12 \mathrm{~V}$ and introducing an adapted $14 \mathrm{~V}$ three-phase ac alternator.

\section{B. Automotive Battery in Present Vehicles}

This technical concept is unchanged to the present day. Figure 2 shows voltage and current measured during cranking of a high-end engine at ambient temperature. The engine is running within about $100 \mathrm{~ms}$. Even at low temperature, a modern car ICE is running within some seconds_-or will not crank at all.

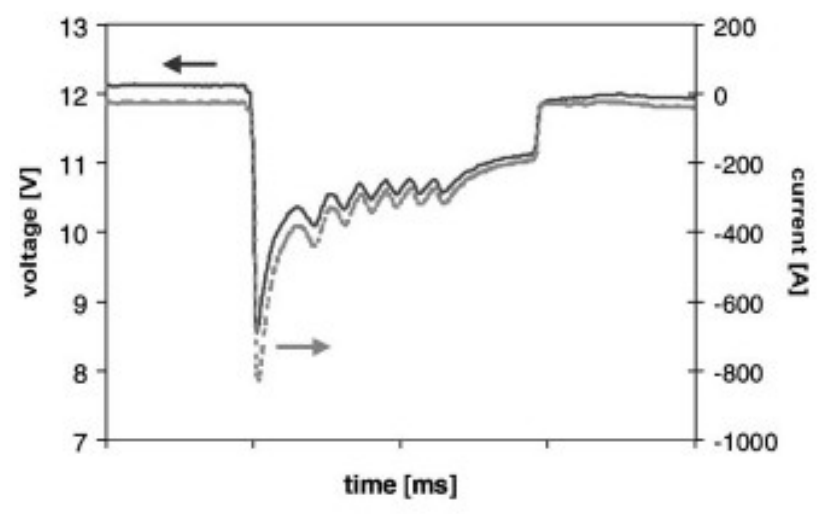

Figure 2. Voltage and current profile during cranking of a high-end gasoline engine at ambient temperature

This so-called partial state-of-charge (PSOC) operating mode is standard for SLI batteries since decades. Typical SOC levels are about $90 \%$ after an extended highway drive in summertime, down to less than $50 \%$ in a traffic jam condition in wintertime-or even much less, which may generate cranking problems when the engine is switched off in this state. 
The actual recharge voltage at the battery terminals depends on the actual alternator voltage and on the Ohmic losses at their connection, according to the current flowing to or from the battery or to other components. This may reduce the battery recharge voltage by several $100 \mathrm{mV}$ compared to the alternator output voltage as can be measured with upper-class vehicles with the battery mounted in the trunk. Even if a temperature-dependent voltage regulator is used, this is mounted near to the alternator, and does not care about the battery temperature which may still be low for hours when the alternator is already at operational temperature. There is no control of recharge current, and the state-of-charge of the battery is a scarcely predictable function of electrical loads, driving conditions, alternator, and regulator properties, and battery properties including size, design, temperature, and battery ageing.

\section{Automotive Battery in Future Vehicles}

Various technological directions for future road vehicles may come up independently or in combinations, depending on the different goals of safety, comfort and economy:

1. Even more components which need electrical power with high-reliability.

2. The demand for “ensured mobility", i.e. cranking and energy supply to essential functions under all (standard or misuse) conditions.

3. Further extension of electrical demand, including new types of electrically driven components with new profiles, including higher (peak) power demand and higher current transients.

4. Start/stop operation mode of the ICE.

5. Electrical brake energy recovery (recuperation).

6. Torque assist/acceleration assist (boost) mode.

The power demand of upper-class vehicles, starting from less than $500 \mathrm{~W}$ in the nineteen sixties, had increased to more than $2 \mathrm{~kW}$ by the year 2000, and will further increase, and will be followed by middle class and compact class vehicles. For the next decade, automotive engineers predict an explosive increase to about $10 \mathrm{~kW}$.

\section{Battery Monitoring}

Battery Monitoring allows for best use of the capability of a battery of given size, to guarantee power supply for high reliability devices, and for replacement strategies. Further-more, monitoring of the actual state-of-charge allows for an electrical power management which may include both reducing consumption of electrical power by limiting of operable luxury applications as well as increase of power generation by appropriate control of alternator or even idle speed and automatic gearbox control.

Battery Monitoring may be needed if

1. Energy has to be provided for a component which is essential for operation, e.g. an Electromechanical Power Steering (EPS) or an Electro-hydraulic Power Braking (EHB) system, an electrically powered suspension stabilisation system, or an automatic gear shift;

2. An Electrical Energy Management (EEM) has to guarantee, e.g. for future cranking capability;

3. The cranking capability has to be supervised to operate a stop/start-system;
4. An indication of battery fatigue is needed for garage service to replace the battery.

Battery Monitoring consists of data acquisition, data processing, and some prediction of the future. For different technical goals, different information with respect to the future is needed. Any approach for Battery Monitoring may be classified according to the following criteria, which may be combined, too, e.g. data acquisition from both long-term and the nearest past, and prediction of both battery status and behavior.

\section{A. Data Acquisition}

1. Type of data: Battery status/battery behavior/operational conditions

2. Time scale of data acquisition: From long-term history/near past

3. Source of data: External battery parameters /internal battery parameters (e.g. electrolyte properties)/vehicle data (e.g. engine rpm, speed, environmental temperature).

4. Data achieved from: Undisturbed battery behavior/ after electrical stimulation.

\section{B. Data analysis}

1. Analysis of operational history (i.e. conditions the battery had to suffer so far).

2. Analysis of previous performance (i.e. behavior the battery has shown so far).

3. Analysis of actual performance (i.e. recent and actual battery behavior and status).

C. Prediction of battery performance under a hypothetical future electrical load

1. Point in time for prediction: Near future (just now, with the present battery status)/medium future (in several hours or days, when the battery charge and temperature may have been changed).

2. Type of predicted battery data: Status (temperature, state-of-charge)/load behavior.

D. Determination of available electrical energy

This is a special case of $\mathrm{C}$, with the standard capacity test scheme as the hypothetical (future) electrical load.

E. Determination of battery degradation (state-ofhealth (SOH) figure of merit).

Figure 3 sketches the interdependence of some of these aspects.

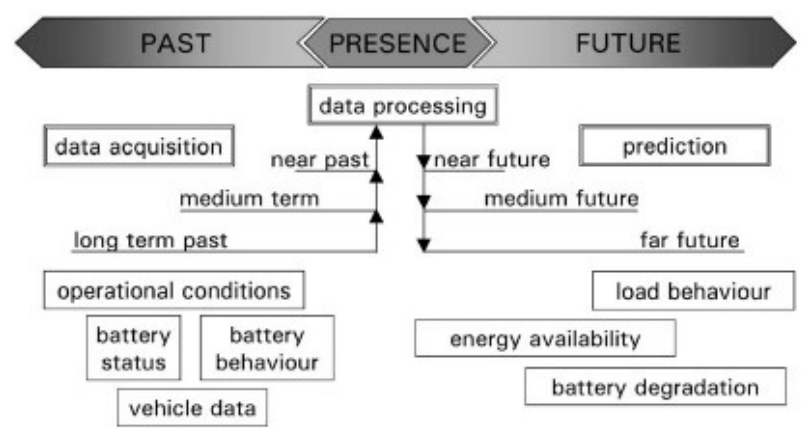

Figure 3. Sketch of interdependence of data acquisition from battery and vehicle, data processing, and prediction with respect to time scale

While Battery Monitoring may provide information about the status of the battery, this knowledge is not a goal by itself. The final technical benefit has to be made clear, and a strategy and means to achieve this goal have to be worked out, to find out the relevant properties of the battery which have to be considered and evaluated. 
Figure 4 sketches qualitatively the dependence of SOF for a discharge load on SOC and $\mathrm{SOH}$ at a given temperature.

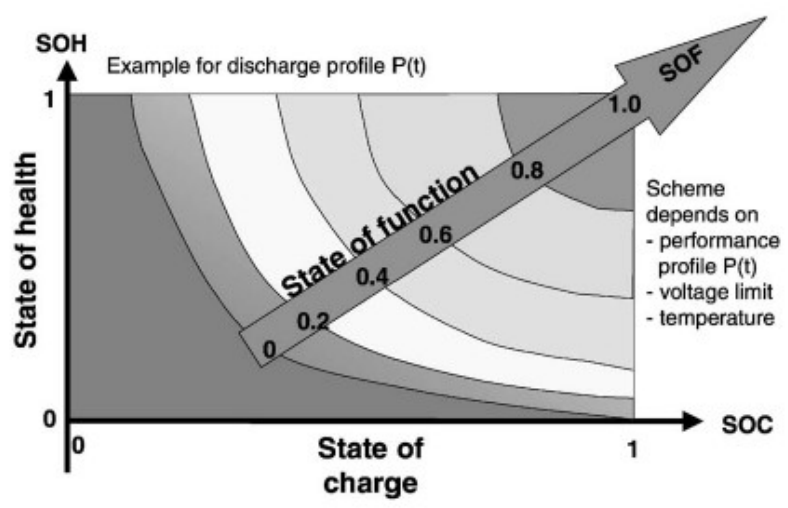

Figure 4. Qualitative dependency of SOF for a discharge load on SOC and $\mathrm{SOH}$ at given $\mathrm{T}$

\section{Approaches for Battery Monitoring}

To analyze the battery status, preferably voltage and current as function of time are evaluated. Determination of temperature is needed to compensate its influence on battery properties. Processing of measured data may be done in various ways, following the alternative concepts,

1. balancing of current flow for tracking of changes of state-of-charge

2. comparison with a characteristic pattern

3. input to a mathematical model, describing the characteristic behavior of the battery

4. estimation of a dynamical battery impedance

5 . valuation of battery voltage at point of zero current

6 . valuation of battery open circuit voltage

All approaches may operate with fixed parameter values, or with parameter values learned from previous operating duty, and need reference values. Balancing of current flow allows for tracking of changes of state-ofcharge, but does not provide an absolute SOC value. Therefore, a reset for calibration of absolute state-ofcharge is needed. Further practical handicaps of current integration over time are the accumulation of sensing and calculation errors over time and the unknown recharge efficiency (recharge factor $>1.0$ ). Therefore, a reset for calibration is needed not only once but periodically. With current integration, only a SOC value can be generated, but no prediction of battery performance SOF at other than nominal test conditions (nominal discharge current and cut-off voltage, nominal temperature), nor any figure for battery ageing $\mathrm{SOH}$ can be made.

Comparison with a characteristic pattern, which may be given as a characteristic diagram or as a functional dependency, is a pattern recognition approach, i.e. knowledge and understanding of battery processes is not essential. The quasi-stationary battery voltage $U$ under current load i is sketched in a characteristic diagram (or mathematical function) as a function of battery temperature $\mathrm{T}$ and SOC. This approach can be applied directly for monitoring of batteries which are being operated mostly under quasi-stationary conditions, i.e. with limited relative current changes.
However, in automotive applications, many current transients and reversals disturb the quasi-stationary state, and voltage cannot be evaluated by comparison with tabled quasi-stationary values. Tabling of transient behaviour is scarcely possible due to the manifold transients. Therefore, a filter function has to detect when the voltage $\mathrm{U}(\mathrm{t})$ has become quasi-stationary after a current transient $\mathrm{i}(\mathrm{t})$, and if the procedure is applicable. In many cases, a voltage U0 (t) filtered by a low frequency (PT1) pass filter (or by a combination of several filters) from $\mathrm{U}(\mathrm{t})$ is evaluated to allow for evaluation sufficiently often.

If characteristic voltages $\mathrm{U}$ are tabled for the whole (i, $\mathrm{T}$, SOC) manifold needed, SOC figures are obtained, and SOF predictions can be made. To obtain information of battery ageing $\mathrm{SOH}$, battery degradation has to be introduced as a further table parameter besides (i, T, SOC), and a procedure has to be provided to distinguish influences from SOC and from SOH.

A mathematical model, describing the characteristic behaviour of the battery, needs to have model parameters correlated to all the battery properties about which information is wanted. As a model, electrical equivalent circuits are favoured comprising electrical components (resistance R, impedance L, capacity C, Warburg term $\mathrm{ZW}$, constant phase element CPE, etc.) describing the battery's internal processes (Figure 5).

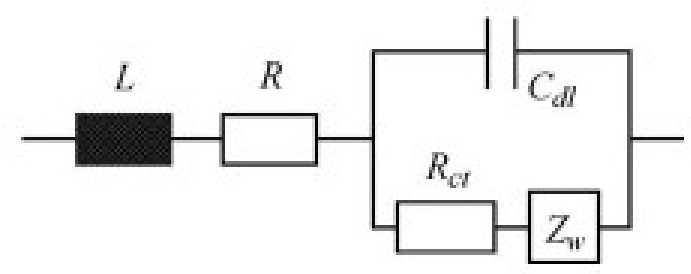

Figure 5. Equivalent circuit for lead-acid battery

Apart from recognition approaches, understanding of the electrochemical processes (e.g. [25]) is essential to assess the validity and to recognise the limits of relevance of appropriate equivalent circuits, which may depend on the operational situation. However, switching of equivalent circuits under operation is a challenging task for numerical stability. To fit the parameters of the equivalent circuit to the measured data, usually algorithms from control theory are used, especially filter functions like the Kalman filter approach.

Parameter fitting is facilitated by changes of the system, i.e. the typical current transients of automotive duty are highly useful. Equivalent circuit approaches both provide SOC figures and allow for predictions of battery performance (SOF) under arbitrary conditions. To include $\mathrm{SOH}$ figures to obtain information of battery degradation, the equivalent circuit has to be extended accordingly. However, this increase of the number of free parameters may harm numerical stability if parameters are strongly correlated.

Estimation of a dynamical battery impedance Rdyn by differentiating measured voltage by current Rdyn $1 / 4$ $\mathrm{dU}=\mathrm{di}$ is mathematically an easy approach. However, the Rdyn value depends not only on battery temperature $\mathrm{T}$ and SOC but also on battery current $i$, as the electrode charge transfer reactions are strongly non-linear. Therefore, filter 
functions are needed to assess Rdyn only if appropriate. Limited sensor precision, ac ripple from the alternator, and electromagnetic interference (EMI) are further hurdles to be overcome.

Dynamical impedance Rdyn allows for prediction of battery load voltage under short load currents, i, which differ not too much from the current with which Rdyn had been determined. This is possible only if battery status has not changed, i.e. for the very near future, while long-term predictions may carry significant deviations, as Rdyn may change rather quickly in highly dynamic battery duty. Some SOC information may be gained from Rdyn using the well-known dependence of electrolyte resistance from SG and SOC, if appropriate reference values are given. However, additional information is needed to distinguish influences from electrolyte resistance from those originating from electrolyte inhomogeneity, grid resistance, etc.

Table 1 summarizes the most important properties of the different approaches and makes clear that a single method will be scarcely sufficient to cover all demands. However, an intelligent combination of several procedures may compensate the mutual weaknesses.

Table 1. Battery Monitoring approaches-summary of key properties

\begin{tabular}{|c|c|c|c|c|c|c|}
\hline$\#$ & Approach & SOC & $\mathrm{SOH}$ & SOF & Pros & Cons \\
\hline 1 & $\begin{array}{l}\text { Balancing of current } \\
\text { flow/current } \\
\text { intergration }\end{array}$ & + & - & - & $\begin{array}{l}\text { Works for discharge and recharge } \\
\text { phases; independent of engine status; } \\
\text { no mathematics; }\end{array}$ & Needs periodical re-set (e.g.by \#6); no information \\
\hline 2 & $\begin{array}{l}\text { Comparison with } \\
\text { characteristic } \\
\text { pattem }\end{array}$ & + & $-/(+)$ & + & $\begin{array}{l}\text { Works for discharge and recharge } \\
\text { phases; limited mathematics; option } \\
\text { for continuous improvements }\end{array}$ & $\begin{array}{l}\text { Large data set for precise operation; hanging of } \\
\text { degradation very difficult; interference by short- } \\
\text { term history } \rightarrow \text { poor in erratic dynamic situations }\end{array}$ \\
\hline 3 & $\begin{array}{l}\text { Mathematical } \\
\text { model/equivalent } \\
\text { circuit }\end{array}$ & + & $(+)$ & + & $\begin{array}{l}\text { Limited data set; very flexible; option } \\
\text { for continuous improvements }\end{array}$ & $\begin{array}{l}\text { Complex equivalent circuits require high } \\
\text { calculation power; learning required } \rightarrow \text { poor in } \\
\text { static situations }\end{array}$ \\
\hline 4 & $\begin{array}{l}\text { Dynamical } \\
\text { impedance } \mathrm{R}_{\text {clyn }}\end{array}$ & - & - & $(+)$ & Limited mathematics & $\begin{array}{l}\text { Prediction for actual SOF status only; no separate } \\
\text { SOC and SOH information; needs many } i=0 \\
\text { situations }\end{array}$ \\
\hline 5 & $\begin{array}{ll}\text { Valuation } & \text { of } \\
\text { voltage point of } \\
\text { zero current }\end{array}$ & $(+)$ & - & $(+)$ & No current sensor (only trigger at $i=0$ ) & $\begin{array}{l}\text { Limited precision; poor in recharge phase; no } \\
\text { degradation information; needs many } i=0 \text { situations }\end{array}$ \\
\hline 6 & $\begin{array}{l}\text { Valuation of open } \\
\text { circuit voltage }\end{array}$ & + & - & - & $\begin{array}{l}\text { Excellent SOC information; limited } \\
\text { mathematics }\end{array}$ & $\begin{array}{l}\text { No degradation information; extended rest periods } \\
\text { required }\end{array}$ \\
\hline
\end{tabular}

+/-: information achieved/not achieved;(+): information only achieved in special situations/for short-term prediction

\section{Data Acquisition}

Data may be collected from

- the battery, especially voltage $\mathrm{U}(\mathrm{t})$, current $\mathrm{i}(\mathrm{t})$ and temperature $\mathrm{T}(\mathrm{t})$ as a function of time

- sensors taking additional information about internal battery properties, and

- the vehicle, e.g. about periods of operation, driving speed, ignition-on information, engine revs (which is proportional to alternator revs), and data bus information providing knowledge about status of components and control units, etc.

While some of these data may be available already in a modern vehicle via a data bus, an additional sensor is required for most of the information. The cost related to additional sensors is the first hurdle to be overcome. All strategies monitoring automotive batteries aim to work with a minimum number of sensors at a minimum of component cost: reduction of need for precision or acquisition frequency is a development goal by itself.

\section{A. Input Data from Battery}

Taking voltage $\mathrm{U}$, current $\mathrm{i}$ and temperature $\mathrm{T}$ of the battery is the most direct monitoring approach and requires no change to the battery. Voltage should be taken as near as possible to the terminals to avoid Ohmic losses, and the current sensor should register the total battery current. For calculation of derived figures like battery impedance, simultaneous acquisition of $U$ and $i$ data is very important for the dynamic vehicle electric system. The frequency of data acquisition depends on the necessity of the algorithm use. Temperature should be taken as direct to the battery as possible. As battery temperature follows rather slowly to changes of the environmental temperature, and internal battery heating can be neglected in most cases, temperature taken near to the battery may be sufficient, possibly being improved by a thermal model of the heat exchange with its environment.

Due to variations of loads and especially the rectification such noise may provide valuable information of the alternator ac current, battery voltage and current is overlaid with a lot of noise signals (cf. Figure 6). Appropriate analogue and/or digital filtering may be necessary to suppress such noise for analysis. However, evaluation of just such noise may provide valuable information.

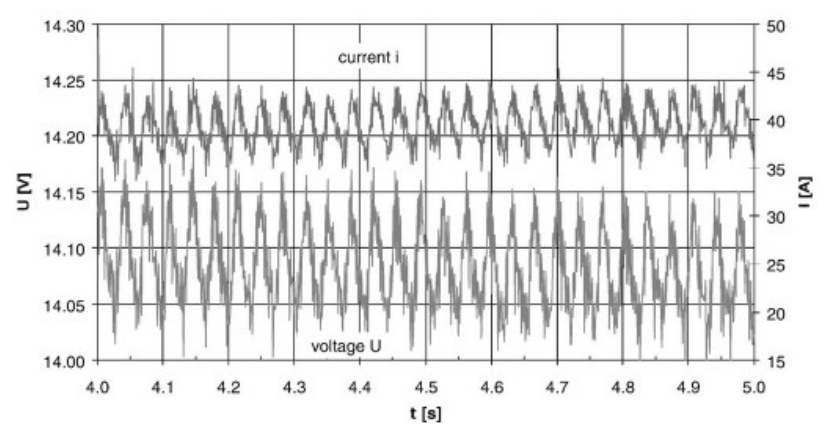

Figure 6. Battery voltage and current overlaid with noise signals from rectification of the alternator ac current; high-end class vehicle with idling engine 


\section{B. Special Sensors}

So far no special sensors integrated into SLI batteries measuring internal properties are established, and at least for lead-acid automotive batteries, this is not expected in the near future.

A temperature sensor within the battery may provide more precise data than sensors attached from the outside or modeling of battery temperature from other temperature information. But as temperature may be inhomogeneous within a battery, the potential benefit of an integrated temperature sensor is limited and has to be critically assessed against cost for this sensor and for reliable data transfer. The same is true for individual cell voltage monitoring or at least for a voltage balance, taking the mid-point voltage of the cell chain. Information about cell imbalance is a valuable indication of improper battery status, which, however, needs combination with means for cell equilibration to provide full benefit.

However, the more dynamics are imposed on the battery, with harsh high-rate cycling operation, with ac ripple loading, and especially with start/stop and boost/recuperation duty, the more benefit may rise from temperature and voltage sensors inside the battery due to imbalances and electrical self-heating as input for battery thermal management and individual cell voltage control, as is done already with hybrid vehicle batteries.

\section{Input Data from Vehicle}

While vehicle data do not directly provide information about battery status, they may be very helpful for valuation of battery data. Ambient and/or engine temperature may help for estimation of battery temperature and of power demand for engine cranking. If the engine is off, battery voltage is not influenced by the alternator, and after the vehicle is locked, the vehicle will go to sleep mode after a limited period of time, with only quiescent currents loading the battery. Such periods are especially suitable for valuation of open circuit voltage. Engine rpm data provide information about alternator capability, and vehicle speed allows for estimation of power demand if electrical power braking or steering components have to be supplied.

\section{Transfer of Input Data}

Analogue data are directed from the sensors to analogue digital converters (ADC), which should be arranged as close as possible to avoid electromagnetic interference (EMI). Generated digital data are directed to the processing unit, which is either arranged nearby, too, or via a digital link. This link is either an individual data bus, or a data bus used also by other components, in which case potential delay in data transfer has to be considered as the Battery Monitoring data are competing with other vehicle data for transfer priority. This may injure real-time capability and synchrony of $\mathrm{U}$ and i data.

\section{E. Data Analysis}

Which type of data from which period of time, at what precision, acquisition frequency, and other criteria, are needed, and what is calculated from these data, depends on the monitoring algorithms, and finally on the type and precision of information needed. All types of algorithm need some information about the battery properties and the load profiles for which performance prediction is requested. If battery type and properties including degradation, and load profiles, are completely fixed, these data can be stored as constants. Otherwise, these values have to be learned while (or before) the Battery Monitoring algorithms operating.

While input data have to be acquired at the location they can be measured with least effort and highest precision, and output data have to be transferred to the location where use shall be made of them, opinions about the best location of the analysing hardware for Battery Monitoring are diverging. If hardware were mounted to the battery side, the battery's characteristic parameters could be stored already upon battery manufacturing, making "burn in" phases and "pairing" of battery with control unit obsolete. On the other hand, load conditions for which performance prediction is requested are given by the system, i.e. the vehicle, and this information would have to be transferred from the vehicle to the control unit at the battery, which means "burn in" phases and/or "pairing" of battery (including the control unit) with the vehicle.

The automotive battery of today is a commodity product, which is replaced not only by qualified personnel in a workshop but also unqualified personnel. It cannot be excluded that a battery different from the unit provided by the vehicle manufacturer is mounted. As any monitoring algorithm uses some characteristic battery information for reference, independent of self-type, procedures are needed to estimate the essential reference data. Reference values, e.g. nominal capacity or Rdyn, may be "learned" during a "burn in" operation phase after battery mounting, when it is in a well-defined state. However, any lack of precision of this state may generate significant consecutive errors.

\section{Battery Degradation}

Battery Monitoring and Management has to consider the change of lead-acid battery properties over life, including grid corrosion, water loss, changes of active material properties, loss of active material availability, etc. Energy throughput, i.e. battery cycling, is gaining in importance with new vehicles, but the dissolution/ precipitation process as the main reaction of the lead-acid system is a fundamental handicap for cycle life for all lead-acid batteries, with both flooded and immobilized electrolytes, and in both prismatic and in spirally wound designs.

Valuation of the actual SOF does not necessarily need to distinguish between short-term (reversible) influences like electrolyte depletion effects, and long-term (irreversible) degradation, e.g. from mass shedding or corrosion, while predictions of battery performance in the medium or far future can be made only if these effects are handled separately. An actual increase of battery impedance helps prediction of reduced short-term high power capability. For prediction of long-term behavior, at another battery status (SOC, T), permanent origins from grid corrosion have to be separated from those from active material loss, as their dependence on SOC and temperature $\mathrm{T}$ is different. And these permanent origins have to be distinguished carefully from momentary concentration polarization effects and especially the influence of SOC and T.

\section{Cross Check and Plausibility}


To avoid unexpected results, the input data to Battery Monitoring, and the figures generated, should to checked for plausibility before they are used, e.g. by Energy Management. Figures may be checked against their range of plausible values, which may depend on parameters like temperature, SOC, or vehicle status.

- With engine off, voltage is scarcely $>13 \mathrm{~V}$, and current cannot flow in the recharge direction.

- Battery voltage and current may change rather quickly, but more or less synchronously.

- Battery temperature, SOC, and SOH cannot change abruptly

- CSC or a parameter comprising grid resistance should correlate with battery duty history, like charge throughput and/or operational temperature.

\section{Energy and Battery Management}

The technical goal of Energy Management is to guarantee the electrical power supply of a component, in all or only in special situations, e.g. with the ICE running or the vehicle moving. Power supply is usually provided by the alternator when the ICE is running and this has to be considered. If the expected voltage under an estimated future load becomes critically low, or the available amount of charge is too low, energy balance may be improved by a decrease of energy consumption or by an increase of energy generation.

Especially the first is very helpful to improve the shortterm energy situation, e.g. to guarantee the power to supply critical loads by reduction or disconnection of nonessential loads, which can be done in a stepwise manner, until all but the real essential components have been switched off.

More comfortable for the user, but technically more ambitious, is an increase of power generation by alternator stimulation (increasing of the generating magnetic field), by triggering of the engine control to increase idle speed, or by increase of the rpm switch points of the automatic gear shift control. Usually, a higher state-of-charge is sought to meet discharge demands. Therefore, Energy Management is closely linked to battery performance and properties like charge acceptance, etc.

Medium term Energy Management considers the situation several hours or days ahead, when the battery state-of-charge and temperature may have been changed. Cranking capability is the main goal in most cases. With an estimated temperature at the point in time under consideration, the power or current profile $\mathrm{P}(\mathrm{t})$ or $\mathrm{i}(\mathrm{t})$, and the minimum voltage $\mathrm{U} 1$ needed to crank the engine can be calculated. From this information, the minimum available amount of energy Qmin necessary to provide this power profile at $\mathrm{U}>\mathrm{U} 1$ can be deduced. This corresponds to a minimum value SOCmin. If the actual SOC is higher than SOCmin, Energy Management may keep house with the excess (SOC À SOCmin) to cover both quiescent loads and additional comfort loads like radio etc. However, if the actual SOC is lower than SOCmin immediate means to increase SOC have to be activated to maintain future cranking capability.

A schematic flow chart for Energy Management to provide cranking capability several days ahead is shown in Figure 7.

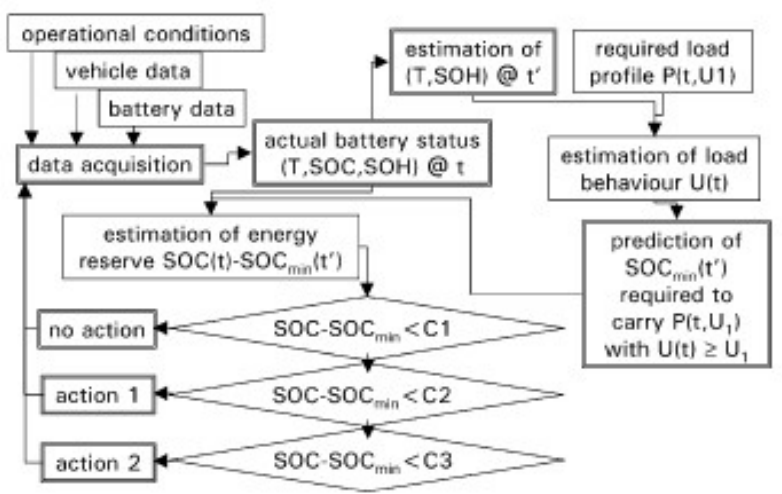

Figure 7. Flow Chart for Battery Monitoring and Power Management to provide cranking capability several days ahead

\section{Conclusion}

Vehicle electric systems will be further driven by fuel economy, ecology, and by new functions for improvement of safety, comfort, and reliability.

Electrically driven components needing electrical power with high-reliability will penetrate the mass market soon, and start/stop systems will reduce ICE idle speed running.

The automotive industry and its suppliers are aiming at solutions comprising the $42 \mathrm{~V}$ PowerNet to be open for technical solutions with very high power demand like new cranking technologies and other components. Standardization of the " $42 \mathrm{~V}$ PowerNet" with the ISO is under way. In advanced "smart hybrid"' designs, the drive modes (ICE only, electric only, both, recharge battery, braking by mechanical brakes or by recuperation, etc.) are chosen by an intelligent drive control system, which considers energy consumption (fuel and electric), emissions and battery status. This includes keeping the battery state-of-charge within an optimal operating window.

In the long-term future, the role of the ICE may be taken by a fuel cell which directly provides electrical energy for driving. This will change but not revolutionize the battery operating conditions, as a fuel cell is normally not able to operate as an electrolyser. So an electrical storage device is needed for peak power shaving, for consumers at stand still, and for recovery of braking energy. However, changes of the vehicle electric power architecture are expected to proceed evolutionary rather than in a revolutionary manner. Due to cost considerations as well as uncertainties with respect to the availability and reliability of newly designed components, modifications will be introduced stepwise only when really needed. This process is expected to last many years.

Battery Monitoring and Energy Management becomes even more essential when new types of duties are imposed on the batteries, changing from a passive component to a pivot unit which has to be monitored, supervised and managed to maintain the vehicle functionality and safety-for both present $14 \mathrm{~V}$ and for future $42 \mathrm{~V}$ PowerNet levels. After high initial enthusiasm, a date for introduction of $42 \mathrm{~V}$ systems is not yet fixed. But there is already demand for Battery Monitoring and Energy Management. Solutions for series vehicles launched in 
years 2001-2003, operating at a $14 \mathrm{~V}$ level, have been developed and are being further improved.

\section{Acknowledgement}

The authors would like to thank B.S. Abdur Rahman University for providing the work environment and the needed support.

\section{References}

[1] T. Keim, "Technical and cost challenges for 42 V implementation', Proceedings of the Papers Presented at 2nd Advanced Automotive Battery Conference, Las Vegas, 2002.

[2] J. Kassakian, "Challenges of the new 42 volt architecture and progress on its international acceptance”, in: VDI Berichte No. 1415, "VDI Verlag, Dusseldorf, 1998, p. 21.

[3] A. Graf (Ed.), in: Proceedings of the Papers Presented at " 1 st International Congress 42 V PowerNet: The First Solutions”, Villach, Austria, 28-29 September 1999.

[4] A. Graf (Ed.), The new automotive $42 \mathrm{~V}$ PowerNet-Preparing for Mass Production, in: 2001 Proceedings of the Papers Presented at the 2nd International Congress on $42 \mathrm{~V}$ PowerNet, Ludwigsburg, Germany, expert-Verlag, Renningen-Malmsheim, 24-25 April 2001.

[5] S. Mueller, X. Pfab, Considerations implementing a dual voltage power network, in: Proceedings of the SAE Convergence Con ference, October 1998.

[6] R. Schoettle, H. Bischof, Das ZweispannungsbordnetzKomponen-ten und Energiemanagement, in [46].
[7] J.P. Douady, C. Pascon, A. Dugast, G. Fossati, Optimized batteries for cars with dual electrical architecture, J. Power Sources 53 (1995) 367-375.

[8] DaimlerChrysler Hightechreport, Vol. 1, 2002, pp. 28-31.

[9] P. Kille, H. Rewald, U. Dierker, J. Kluge, J. Odening, J. Leohold, Das elektrische Bordnetz, VW Phaeton, ATZ and ETZ (Automobiltech- nische Zeitschrift/Motortechnische Zeitschrift), special edition, July 2002, p. 132.

[10] A.-M. Kreipp, M. Zuber, P. Duba, Q. Sterner, 42 V systems and development by simulation, in: A.-M. Kreipp, E. Lodowicks, R. Regler, M. Zuber (Eds.), 2001 Proceedings of the Papers Presented at the 2nd International Congress on $42 \mathrm{~V}$ PowerNet, “'Batterie- und 'Energiemanagement', VDI Berichte 1646, VDI Verlag, Dusseldorf, 2001, p. 495.

[11] D.U. Sauer, G. Bopp, A. Jossen, J. Garche, M. Rothert, M. Wollny, State of charge-what does we really speak about ? in: Proceedings of the 21st International Telecommunications Energy Conference Presented at INTELEC’99, Poster 31-32, Kopenhagen, 1999.

[12] H.P. Schoener, T.J. Dougherty, State-of-charge, state-of-health, and what system designers really want to know about a battery, in: Proceedings of the Presentation at Meeting of MIT Industry Consortium on Advanced Automotive Electrical/Electronic Compo-nents and Systems, Nagoya, October 2000.

[13] E. Karden, S. Buller, W.W. De Doncker, A method for measurement and interpretation of impedance spectra for industrial batteries, J. Power Sources 85 (2000) 72-78.

[14] E. Karden, "Using low-frequency impedance spectroscopy for characterisation, monitoring and modelling of industrial batteries", Dissertation Thesis, Technical University of Aachen, Germany, 2001.

[15] J.E.B. Randles, Kinetics of rapid electrode reactions, Discuss. Faraday Soc. 1 (1947) 11-19. 